\title{
Adverse effects of antidepressants during pregnancy
}

\author{
Toshi A Furukawa
}

Departments of Health Promotion and Human Behavior and of Clinical Epidemiology, Kyoto University Graduate School of Medicine/ School of Public Health, Kyoto, Japan

Correspondence to Toshi A Furukawa, furukawa@kuhp.kyoto-u.ac.jp

\section{INTRODUCTION}

This is the fourth of a series of evidence-based case conferences. The main aim of this new series of papers is to help clinicians learn and apply the evidence-based approach in their own clinical practices. The clinical question that we seek to solve in each of this series emanates from a real clinical case. I have completely anonymised the scenario but I still hope it remains as real as it was. The presentation in this series may appear too brief and rushed but this is how EBM can be practiced by busy clinicians once you are familiar with the process. This issue's clinical question is about tolerability of drug treatments in a special population (pregnant women). The case conference below illustrates how to critically appraise and apply an article about adverse events. We anticipate that the next case conference (fifth of the series) will focus on how to use a systematic review for a treatment decision.

\section{CLINICAL CASE}

Patient

A 32-year-old woman.

\section{Present illness}

The patient suffered from her first major depressive episode after giving birth to her first baby at the age of 25 . The episode lasted for 2 months and subsided without any treatment. However, when she had her second baby at the age of 28, depression recurred and this time she visited her family doctor for treatment. She was prescribed sertraline $50 \mathrm{mg}$ /day, to which she responded well. However, taking care of two small boys at home was not easy and she had never fully recovered from her second depressive episode. Her mother was diagnosed with malignancy and a third major depressive episode set in. Patient's dose of sertraline was gradually increased to $150 \mathrm{mg} /$ day over a period of 2 months with some benefit and was kept at that level since then.

\section{Present status}

On the maintenance treatment with sertraline $150 \mathrm{mg} /$ day, she was subsyndromally depressive with residual fatigability and loss of confidence. However, she managed to remain relatively functional in her role as mother and wife. The family doctor saw her every 6 weeks. At one of such planned visits, she appeared anxious and told the doctor that she was pregnant again. She had visited an obstetrician, who confirmed that antidepressants overall do not seem to be associated with increased risk of congenital malformations, except possibly for cardiovascular ones. ${ }^{1}$ That night she had a good discussion with her husband, who said that he would ultimately value her judgement but that he himself was against abortion and was willing to help her even more should they decide to continue with the pregnancy. She now asks for more information about the effects of antidepressants during pregnancy and cardiovascular malformations such as ventricular septal defects, atrial septal defects, teratology of Fallot, etc.

\section{FORMULATE YOUR CLINICAL QUESTION}

Patients: Pregnant women with depression

Intervention: Antidepressants

Comparison: No treatment/placebo

Outcomes: Cardiovascular malformation

\section{LITERATURE SEARCH}

Searching for strong evidence on adverse effects is often not straightforward, because ethically randomised experiments cannot be conducted to examine causation of bad outcomes (information about harms in randomised controlled trials is often underpowered, sketchy and unsystematic) and therefore we often have to rely on observational studies for the necessary information. ${ }^{2}$ Searching for high-quality observational studies and then critically appraising them is not easy.

One good portal for high-quality observational studies is PubMed Clinical Queries. You select Category: Etiology with Scope: Narrow (if there appear to be too many candidates) or Scope: Broad (if there appear to be too few). The MeSH terms for pregnancy is 'Pregnancy' and that for antidepressants is 'Antidepressive agents'; entering both and selecting Etiology-Narrow, you immediately noticed that there was one very recent large study on this clinical question: Huybrechts $K F$, Palmsten K, Avorn J, et al. Antidepressant use in pregnancy and the risk of cardiac defects. N Engl J Med 2014;370:2397-407. 


\section{CRITICAL APPRAISAL OF THE LITERATURE ON HARM}

\section{ARE THE RESULTS AT RISK OF BIAS?}

Were patients similar for prognostic factors known to be associated with the outcome (or was statistical adjustment done)?

YES. The study was a cohort study nested in the nationwide Medicaid database and included approximately one million pregnant women. The crude comparison between pregnant women taking antidepressants and those not taking them were statistically adjusted first for the presence of depression diagnosis, and further with propensity scores representing depression severity and other possible confounders.

Were the circumstances and methods for detecting the outcome similar?

YES. The data came from the nationwide database. The surveillance for cardiac malformations may have been more intense for women taking antidepressants but this factor too would have been statistically adjusted as above.

Was the follow-up sufficiently complete?

YES. The outcome (cardiac malformations) was taken from the maternal and infant records during the first 90 days after delivery. Major malformations should have been recorded.

\section{WHAT ARE THE RESULTS?}

What is the risk ratio or $\mathrm{OR}$ and its $95 \% \mathrm{Cl}$ ?

The unadjusted OR of 1.25 (95\% Cl 1.15 to 1.36$)$ became 1.02 $(95 \% \mathrm{Cl} 0.90$ to 1.15$)$ in the analyses restricted to women with depression and stratified by propensity scores. There was no substantial difference in estimated ORs among different antidepressants.
What is the corresponding number needed to harm (NNH) and its $\mathrm{Cl}$ ?

The control event rate for cardiac malformations was 6403 affected infants among 885115 women with no antidepressant exposure, or $0.073 \%$. The $\mathrm{OR}$ above would then correspond with NNH of 5500 ( $95 \% \mathrm{Cl} 3800$ to 9100 ) for the crude comparison, and $69000(95 \% \mathrm{Cl}-\infty$ to $-14000,9000$ to $\infty)$ for the adjusted comparison.

\section{HOW CAN I APPLY THE RESULTS TO PATIENT CARE?}

Is your patient so different from those included in the study that the results may not apply?

The study cohort was around 25 years of age, and mostly Caucasian; on the other hand, the patient in the case vignette is 32 and Japanese. However, the study showed no effect of age or race on the association between antidepressant exposure and malformations. We can therefore expect a similar $\mathrm{OR}$ and NNH for this patient.

Are there important differences in exposures (dose, duration, etc) for your patients?

The patient is on a relatively high dose of sertraline. However, according to the current study there was no dose response relationship between antidepressants and cardiac malformations (as one would expect if the compound itself is not harmful).

What is the balance between benefits and harms for patients like yours?

The patient suffers from recurrent depression which is currently in partial remission on a relatively high dose of sertraline.

Stopping the maintenance antidepressant doubles the risk of recurrence $^{3}$ : for this recurrent, only partially remitted woman

\section{WHAT WILL YOU DO WITH YOUR PATIENT?}

You explain to the patient that there are strong data to suggest that antidepressants overall and sertraline in particular are not associated with increased risk of cardiac malformations in the newborns. The theoretically maximum harm could be one additional malformation per 9000 pregnant women taking antidepressants. You also provide additional information surrounding use of antidepressants during pregnancy. ${ }^{4}$ On the other hand, continuing the antidepressant may decrease the possibility of depression recurrence from $80-60 \%$ to $40-30 \%$.

The patient and her husband came back a week later, declaring that they had decided to continue with the pregnancy and the antidepressant. You now discuss with the couple how they can work together to minimise the risk of recurrence during and after the pregnancy, and also the possible effects of antidepressants on the neonate on delivery. ${ }^{5}$

Competing interests TAF has received lecture fees from Eli Lilly, Meiji, Mochida, MSD, Pfizer and Tanabe-Mitsubishi, and consultancy fees from Sekisui and Takeda
Science Foundation. He is diplomate of the Academy of Cognitive Therapy. He has received royalties from Igaku-Shoin, Seiwa-Shoten and Nihon Bunka Kagaku-sha. The Japanese Ministry of Education, Science, and Technology, the Japanese Ministry of Health, Labor and Welfare, and the Japan Foundation for Neuroscience and Mental Health have funded his research projects.

doi:10.1136/eb-2014-101964

\section{REFERENCES}

1. Grigoriadis S, VonderPorten EH, Mamisashvili L, et al. Antidepressant exposure during pregnancy and congenital malformations: is there an association? A systematic review and meta-analysis of the best evidence. J Clin Psychiatry 2013;74:e293-308.

2. Barbui C, Ostuzzi G. Beneficial and harmful consequences of prepartum and postpartum antidepressant exposure. Evid Based Ment Health 2014;17:69-71.

3. Geddes JR, Carney SM, Davies C, et al. Relapse prevention with antidepressant drug treatment in depressive disorders: a systematic review. Lancet 2003;361:653-61.

4. Einarson A. Antidepressant use during pregnancy: navigating the sea of information. Can Fam Physician 2013;59:941, 943-4.

5. Grigoriadis S, VonderPorten EH, Mamisashvili L, et al. The effect of prenatal antidepressant exposure on neonatal adaptation: a systematic review and meta-analysis. J Clin Psychiatry 2013;74:e309-20. 